\title{
Efetividade da educação em saúde na prevenção da retinopatia diabética
}

\begin{abstract}
RESUMO
Objetivou-se analisar a efetividade da educação em saúde como estratégia para prevenção da retinopatia diabética, mediante revisão sistemática realizada nas bases de dados eletrônicas estabelecidas, sem restrição de idioma e de período de publicação. Incluíram-se estudos do tipo ensaio clínico randomizado, quase-experimental e observacional, e excluíram-se as publicações com delineamento impreciso. Foram analisados cinco artigos que atenderam os critérios de inclusão estabelecidos. A otimização da taxa dos cuidados habituais com os olhos por pessoas com diabetes, após intervenções educativas, variou, após 1 ano, de $61 \%$ para $70 \%(p<0,001)$ e, após 2 anos, de $52 \%$ para $70 \%$ ( $<<0,001)$, com 2,5 a 4,3 vezes mais probabilidade de um indivíduo realizar o exame de fundo de olho em comparação àqueles que apenas refletem sobre sua condição. As evidências apontaram a educação em saúde como eficaz na prevenção e no controle da retinopatia diabética.
\end{abstract}

DESCRITORES: Educação em Saúde; Diabetes Mellitus; Retinopatia Diabética.

\section{ABSTRACT}

The aim of this study was to analyze the effectiveness of health education as a strategy for the prevention of diabetic retinopathy, through a systematic review conducted in the established electronic databases, without restriction of language and publication period. Studies of the randomized, quasi-experimental and observational clinical trial type were included, and publications with inaccurate design were excluded. We analyzed five articles that met the inclusion criteria established. The optimization of the standard eye care rate for people with diabetes after educational interventions ranged from $1 \%$ to $70 \%$ after 1 year $(p<0.001)$ and after 2 years from $52 \%$ to $70 \%(p<0.001)$, with 2.5 to 4.3 times more likely for an individual to have an eye exam compared to those who only reflect on their condition. Evidence pointed to health education as effective in preventing and controlling diabetic retinopathy. KEYWORDS: Health Education; Diabetes Mellitus; Diabetic Retinopathy.

\section{RESUMEN}

El objetivo de este estudio fue analizar la efectividad de la educación sanitaria como estrategia para la prevención de la retinopatía diabética, a través de una revisión sistemática realizada en las bases de datos electrónicas establecidas, sin restricción de idioma y período de publicación. Se incluyeron estudios del tipo de ensayo clínico aleatorizado, cuasi-experimental y observacional, y se excluyeron las publicaciones con diseño inexacto. Analizamos cinco artículos que cumplían los criterios de inclusión establecidos. La optimización de la tasa de atención ocular estándar para personas con diabetes después de intervenciones educativas varió del $1 \%$ al $70 \%$ después de 1 año $(p<0,001)$ y después de 2 años del $52 \%$ al $70 \%$ ( $<<0.001)$, con 2.5 a 4.3 veces más probabilidades de que un individuo realice el examen de fondo de ojo en comparación con aquellos que solo reflexionan sobre su condición. La evidencia apunta a que la educación para la salud es efectiva para prevenir y controlar la retinopatía diabética.

PALABRAS CLAVE: Educación en Salud; Diabetes Mellitus; Retinopatía Diabética.

RECEBIDO EM: 09/11/2019 APROVADO EM: 11/11/2019

\section{Natalia Daiana Lopes de Sousa}

Enfermeira. Doutoranda do Programa de Pós-Graduação Cuidados Clínicos em Enfermagem e Saúde. Universidade Estadual do Ceará. Fortaleza, CE, Brasil.

\section{Eline Saraiva Silveira Araújo}

Enfermeira. Doutoranda do Programa de Pós-Graduação Cuidados Clínicos em Enfermagem e Saúde. Universidade Estadual do Ceará. Fortaleza, CE, Brasil. 


\section{artigo}

Sousa, N.D.L.; Araújo, E.S.S.; Falcão, L.M.; Vieira, L.A.; Sousa, M.L.; Pereira, C.S. Guedes, M.V.C.;

Efetividade da educação em saúde na prevenção da retinopatia diabética

\section{Lariza Martins Falcão}

Enfermeira. Doutoranda do Programa de Pós-Graduação Cuidados Clínicos em Enfermagem e Saúde. Universidade Estadual do Ceará. Fortaleza, CE, Brasil.

\section{Luara Abreu Vieira}

Enfermeira Doutoranda do Programa de Pós-Graduação Cuidados Clínicos em Enfermagem e Saúde. Universidade Estadual do Ceará. Fortaleza, CE, Brasil.

\section{Maria Lucilândia de Sousa}

Graduanda de Enfermagem. Universidade Regional do Cariri. Crato, CE, Brasil.

\section{Camila da Silva Pereira}

Graduanda de Enfermagem. Universidade Regional do Cariri. Crato, CE, Brasil.

\section{Maria Vilani Cavalcante Guedes}

Enfermeira. Doutora. Docente do Programa de Pós-Graduação Cuidados Clínicos em Enfermagem e Saúde. Fortaleza, CE, Brasil.

\section{INTRODUÇÃO}

A retinopatia diabética $(\mathrm{RD})$ é uma complicação microvascular altamente específica do diabetes mellitus (DM) tipos 1 e 2, com prevalência fortemente relacionada à duração da doença e ao nível de controle glicêmico $^{(1)}$. É classificada mediante a avaliação do fundo ocular/retinografia e subdivide-se em dois estágios principais: não proliferativa (RDNP) e proliferativa (RDP). Em sua fase precoce, a RDNP caracteriza-se por microaneurismas, hemorragias e exudatos rígidos, podendo evoluir com surgimento de áreas isquêmicas. A RDP destaca-se pela presença de neovascularização ${ }^{(2)}$.

Existem, aproximadamente, 93 milhões de indivíduos no mundo com algum grau de $\mathrm{RD}$, e um terço desta população necessita de algum tipo de tratamento. A RD é uma das principais causas de cegueira evitável em pessoas com idade entre 16 e 64 anos, afetando cerca de 35 a $40 \%$ das pessoas com DM - aproximadamente 4 milhões de pessoas no Brasil. Estima-se que mais de $90 \%$ das pessoas com DM tipo 1 e $60 \%$ com o tipo 2 devem apresentar algum grau dessa complicação após 20 anos da doença, porém, devido ao caráter assintomático do $\mathrm{DM}$, recomenda-se a avaliação oftalmológica imediatamente após o diagnóstico ${ }^{(3)}$.

A educação em saúde tem impacto positivo no comportamento das pessoas com DM, mediante mudanças no estilo de vida, autogerenciamento, controle glicêmico e, consequente, equilíbrio metabólico, refletindo no controle da evolução da doença e na prevenção de complicações agudas e crônicas, permitindo que os indivíduos sejam corresponsáveis por sua saúde, mediante participação ativa ${ }^{(4)}$.

Os cuidados clínicos de enfermagem de pessoas com DM são preponderantes em meio ao processo de prevenção das complicações advindas desta condição crônica e, dentre eles, destaca-se o enfermeiro como educador e promotor de saúde, contribuindo no incentivo ao autocuidado, no acréscimo de conhecimentos, reflexões e mudanças no estilo de vida, à troca de experiências, à melhor convivência com pessoas com DM e à aceitação emocional ${ }^{(5,6)}$.

Considerando a necessidade de mais in- formações acerca do assunto, objetivou-se analisar as evidências científicas da efetividade da educação em saúde como estratégia para prevenção da $\mathrm{RD}$. A pergunta norteadora foi: Qual a efetividade da educação em saúde na prevenção/controle da RD?

\section{METODOLOGIA}

Trata-se de revisão sistemática da literatura que obedeceu as seguintes etapas: elaboração da pergunta de pesquisa; busca na literatura; seleção dos artigos; extração dos dados; avaliação da qualidade metodológica; síntese dos dados; avaliação da qualidade das evidências; e redação e publicação dos resultados ${ }^{(7)}$.

Para a elaboração da pergunta de pesquisa, foi utilizada a estratégia PICOS, no qual "P", de Population, corresponde qual a população em foco; "I", de Intervention, define qual o tipo de intervenção estudado; "C", de Comparison, identifica o grupo controle que será testado juntamente da intervenção; "O", de Outcome, indica os desfechos avaliados; e "S", de Study type, identifica o tipo de estudo(7) - Quadro 1.

Quadro 1. Acrônimo PICOS utilizado para a busca de dados. Fortaleza, CE, Brasil, 2018.

\begin{tabular}{|l|c|l|}
\multicolumn{1}{|c|}{ DESCRIÇÃO } & ABREVIAÇÃo & \multicolumn{1}{c|}{ COMPONENTES DA PERGUNTA } \\
\hline População & $\mathrm{P}$ & Pessoas com diabetes mellitus tipos 1 ou 2 \\
\hline Intervenção & $\mathrm{I}$ & Educação em saúde \\
\hline Comparação & $\mathrm{C}$ & Sem comparação \\
\hline Desfecho & $\mathrm{O}$ & Prevenção/controle da retinopatia diabética \\
\hline Tipo de estudo & $\mathrm{S}$ & $\begin{array}{l}\text { Estudos observacionais (relato de caso, série de caso, caso-controle, coorte e transversal) } \\
\text { Estudos experimentais (ensaios clínicos) Estudos quase-experimentais }\end{array}$ \\
\hline
\end{tabular}


Para a busca na literatura, os descritores foram cruzados, de acordo com as particularidades de cada base de dados, sendo obtidos por consulta aos Descritores de Ciências em Saúde (DeCS) "promoção da saúde", "educação em saúde", "retinopatia diabética" e "complicações do diabetes" e seus respectivos Medical Subject Headings $(\mathrm{MeSH})$, conectados pelos operadores booleanos "AND” e "OR". A estratégia de busca foi: "health promotion" "OR" "health education" "AND" "diabetic retinopathy" "OR" "diabetes complications".

A busca ocorreu em abril de 2018 nas seguintes bases de informação: PubMed, Cumulative Index to Nursing and Allied Health Literature (CINAHL), Web of
Science, Scopus e Biblioteca Virtual em Saúde (BVS).

Após a etapa de busca, foram selecionados estudos a partir da revisão dos títulos e resumos, de acordo com os seguintes critérios para inclusão: artigos originais disponíveis na íntegra com delineamento metodológico do tipo ensaio clínico randomizado, quase-experimental e observacional e que abordassem a questão de pesquisa. Em seguida, realizou-se a leitura dos textos completos, buscando eleger os estudos que respondessem à pergunta de investigação. Após esse processo, foram excluídas as publicações com delineamento impreciso e os artigos que estavam em duplicata foram contados apenas uma vez (Figura 1).

Figura 1. Fluxo de seleção dos artigos primários para a revisão sistemática, adaptado do modelo PRISMA(8). BVS: Biblioteca Virtual em Saúde; CINAHL: Cumulative Index to Nursing and Allied Health Literature. Fortaleza, CE, Brasil, 2018

Artigos selecionados na busca primária para a leitura do título e resumo
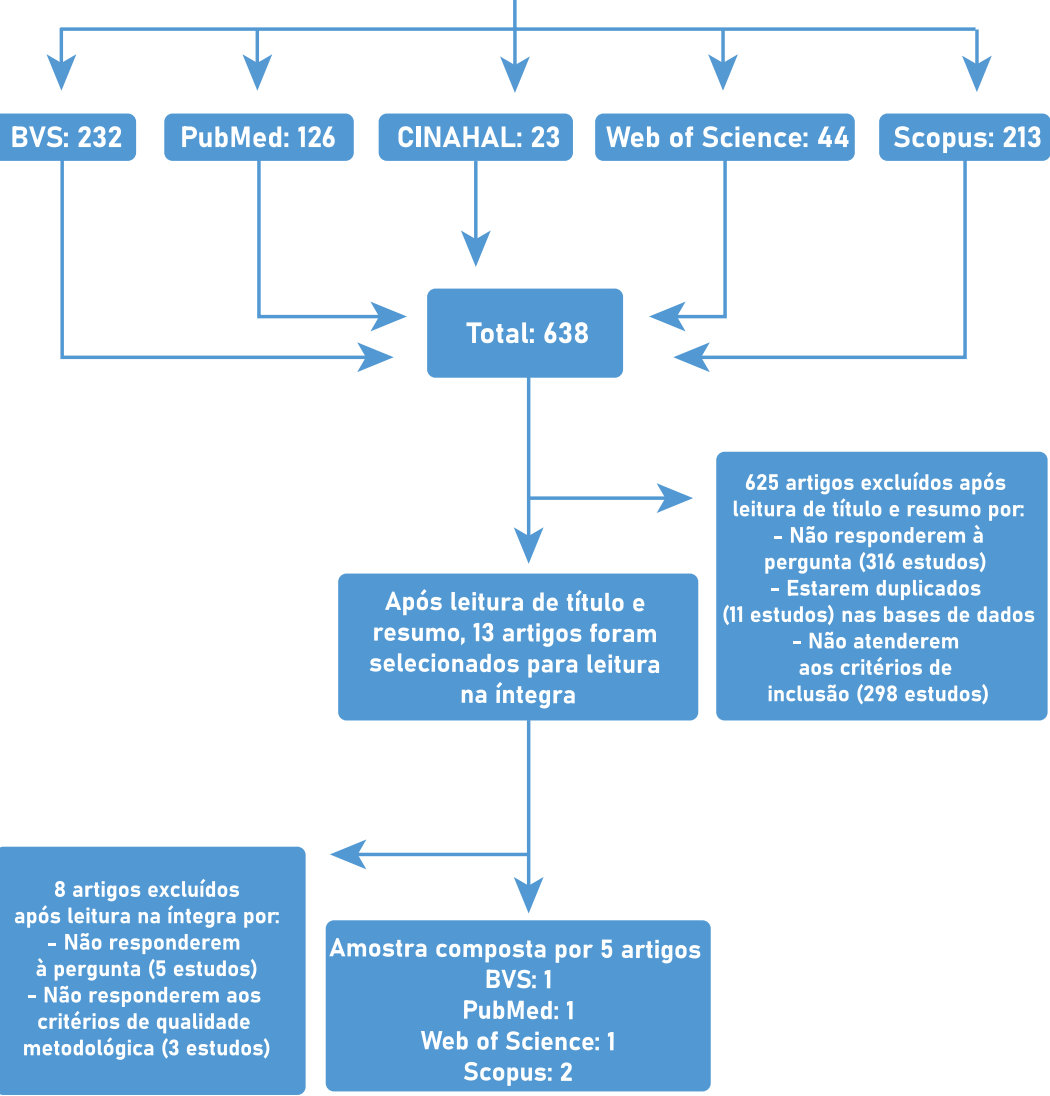

Para a avaliação da qualidade metodológica dos estudos observacionais, utilizou-se o checklist Strengthening the Reporting of Observational Studies in Epidemiology (STROBE), em que cada item recebeu uma pontuação de zero e 1 , sendo zero para não relatado e 1 para relatado. Foram excluídos os artigos que não atingiram $50 \%$ dos critérios $^{(9)}$.

Para a avaliação dos ensaios clínicos randomizados, utilizou-se a escala de Jadad, que consiste em itens direcionados à redução de viés de pesquisa (randomização, cegamento e destino de todos os participantes), somando até 5 pontos. Estudos em que a pontuação foi menor do que 3 foram excluídos, por serem classificados de má qualidade ${ }^{(10)}$.

Para os estudos quase-experimentais, a avaliação foi realizada mediante lista de verificação de medição de qualidade estabelecida, composta por 27 perguntas com respostas "sim" ou "não" ou "parcialmente" ou "incapaz de determinar", dividida em cinco partes que correspondem a: qualidade do estudo (dez itens), validade externa (três itens), avaliação de viés relacionado à intervenção e medida de desfecho (sete itens), determinação de viés da amostragem ou atribuição de grupo (seis itens) e poder do estudo (um item). O ponto de corte utilizado para considerar os estudos de boa qualidade foi superior a $50 \%$ da pontuação máxima ${ }^{(11)}$.

Os estudos foram selecionados mediante dupla leitura. Neste processo, primeiramente, foi realizada a seleção das publicações relevantes, tendo sido aplicados os critérios de inclusão e exclusão; em seguida, foi feita a seleção das publicações, a partir dos critérios de qualidade; e, por fim, realizou-se a escolha dos dados importantes.

\section{RESULTADOS}

Entre os artigos analisados, identificou-se que quatro eram experimentais do tipo ensaio clínico controlado randomizado ${ }^{(12-15)} \mathrm{e}$ um era quase-experimental ${ }^{(16)}$. Houve predomínio de estudos norte-americanos ${ }^{(12-15)}$, seguidos por um australiano ${ }^{(16)}$.

Os estudos apresentaram quatro for- 


\section{artigo}

Sousa, N.D.L.; Araújo, E.S.S.; Falcão, L.M.; Vieira, L.A.; Sousa, M.L.; Pereira, C.S. Guedes, M.V.C.;

Efetividade da educação em saúde na prevenção da retinopatia diabética

mas de intervenções para promover a educação em saúde às pessoas com DM, com objetivo de prevenir ou controlar a RD: materiais impressos como livretos, relatórios e gráficos de risco ${ }^{(12,14,15)}$; $\operatorname{vídeos}^{(12)}$; contatos telefônicos ${ }^{(12,13)}$ e campanhas em meios de comunicação como TV, rádio e jornais ${ }^{16)}$. Todas as intervenções educativas foram feitas por médicos.

O Quadro 2 apresenta a categorização dos achados sobre o tipo de estudo, o método (amostra, intervenção e duração da intervenção), as principais conclusões dos autores, o nível de evidência e o instrumento empregado para avaliar a qualidade.

\section{DISCUSSÃO}

De acordo com a análise da qualidade dos estudos encontrados, nota-se elevada evidência científica devido à predominância de ensaios clínicos controlados randomizados, os quais apresentam o delineamento mais adequado e confiável para questões relacionadas à intervenção ${ }^{(17)}$.

Nas publicações avaliadas, as intervenções educativas foram realizadas mediante material impresso, vídeo, contato telefônico e campanhas em meios de comunicação em massa, porém o uso da intervenção educativa com gráficos de risco, relatórios e material suplementar foi considerado impróprio ${ }^{(15)}$. A otimização do controle glicêmico, a redução da hemoglobina glicada (HbAlc) e a

\section{Quadro 2. Caracterização dos estudos. Fortaleza, CE, Brasil, 2018.}

\begin{tabular}{|c|c|c|c|c|c|c|}
\hline ESTUDO & $\begin{array}{l}\text { TIPO DE } \\
\text { ESTUDO }\end{array}$ & AMOSTRA & INTERVENÇÃO & $\begin{array}{l}\text { DURAÇÃO DA } \\
\text { INTERVENÇÃO } \\
\text { (MESES) }\end{array}$ & DESFECHO & $\begin{array}{l}\text { INSTRUMENTO } \\
\text { DE AVALIAÇÃO/ } \\
\text { PONTUAÇÃO }\end{array}$ \\
\hline Basch et al.12 & $\begin{array}{l}\text { Ensaio clínico } \\
\text { randomizado } \\
\text { controlado }\end{array}$ & 280 & $\begin{array}{l}\text { Livreto colorido; } \\
\text { vídeo motivacional; } \\
\text { educação e } \\
\text { telefonemas } \\
\text { personalizados }\end{array}$ & 6 & $\begin{array}{l}\text { Aumento substancial } \\
\text { das taxas de exame } \\
\text { para rastreio das } \\
\text { complicações } \\
\text { oftálmicas do diabetes }\end{array}$ & $\begin{array}{l}\text { Escala de } \\
\text { Jadad/3 }\end{array}$ \\
\hline Walker et al.13 & $\begin{array}{l}\text { Quase- } \\
\text { experimental }\end{array}$ & 1.700 & $\begin{array}{l}\text { Campanha educativa } \\
\text { (televisão, rádio e } \\
\text { jornais) }\end{array}$ & 12 & $\begin{array}{l}\text { Melhora na utilização de } \\
\text { serviços oftalmológicos }\end{array}$ & $\begin{array}{l}\text { Escala Downs e } \\
\text { Black/54,5\% }\end{array}$ \\
\hline Aiello et al.14 & $\begin{array}{l}\text { Ensaio clínico } \\
\text { randomizado } \\
\text { controlado }\end{array}$ & 598 & $\begin{array}{l}\text { Telefonemas } \\
\text { personalizados }\end{array}$ & 6 & $\begin{array}{c}\text { Melhora significativa na } \\
\text { realização dos exames } \\
\text { de fundo de olho para a } \\
\text { triagem da retinopatia } \\
\text { diabética }\end{array}$ & $\begin{array}{l}\text { Escala de } \\
\text { Jadad/3 }\end{array}$ \\
\hline Weiss et al.15 & $\begin{array}{l}\text { Ensaio clínico } \\
\text { randomizado } \\
\text { controlado }\end{array}$ & 1.482 & $\begin{array}{l}\text { Gráfico de risco, } \\
\text { relatório de risco } \\
\text { personalizado, } \\
\text { avaliação da doença } \\
\text { renal e da retinopatia } \\
\text { e materiais } \\
\text { suplementares de } \\
\text { educação em gestão } \\
\text { do diabetes }\end{array}$ & 24 & $\begin{array}{c}\text { A otimização do } \\
\text { controle glicêmico, } \\
\text { a redução da } \\
\text { hemoglobina glicada e } \\
\text { a melhora dos cuidados } \\
\text { habituais com os olhos } \\
\text { foi modesta em relação } \\
\text { ao grupo controle }\end{array}$ & $\begin{array}{l}\text { Escala de } \\
\text { Jadad/3 }\end{array}$ \\
\hline Müller et al.16 & $\begin{array}{l}\text { Ensaio clínico } \\
\text { randomizado } \\
\text { controlado }\end{array}$ & 206 & $\begin{array}{l}\text { Documentos } \\
\text { impressos }\end{array}$ & 44 & $\begin{array}{l}\text { Aumento significativo } \\
\text { das taxas de exames de } \\
\text { fundo de olho }\end{array}$ & $\begin{array}{l}\text { Escala de } \\
\text { Jadad/5 }\end{array}$ \\
\hline
\end{tabular}


melhora dos cuidados habituais com os olhos não foram estatisticamente satisfatórias no grupo intervenção, sugerindo a investigação de outros paradigmas intervencionais.

O livreto colorido, sucinto e didático, foi direcionado para as pessoas com baixa alfabetização e abordava a definição e características da RD e como se conviver com ela, além de incentivar as pessoas com DM a realizar o exame de fundo de olho pelo menos uma vez a cada ano. $\mathrm{O}$ vídeo educativo usou apelos emocionais por meio da narração de histórias, com o objetivo de aumentar a motivação para realização do exame oftalmológico a cada ano. Já o contato telefônico guiado por um protocolo semiestruturado oferecia educação e aconselhamento individualizado e interativo, além de identificar e compreender as razóes de cada sujeito e/ ou barreiras para realizar um exame de fundo de olho ${ }^{(12)}$.

Outro estudo ${ }^{(14)}$, que também realizou intervenção telefônica, teve a finalidade de educar e motivar as pessoas sobre a importância de se fazer um exame anual de fundo de olho e eliminar as barreiras para a realização desse exame. Comunicações de risco, como a frequente falta de sintomas de $\mathrm{RD}$ e o fato de o tratamento precoce diminuir o risco de cegueira, foram incluídas. Os intervencionistas usaram abordagens de resolução de problemas para capacitar os pacientes a superar barreiras pessoais, motivacionais e institucionais que os impediam de realizar esses exames.

As campanhas educativas (televisão, rádio e jornais), tinham como principais temas prevenção da perda de visão; orientação para alterações na visão em pessoas com mais de 50 anos ou com histórico familiar de doença ocular ou DM; e estimulo à realização de exames oftalmológicos regulares. A proporção estimada da população-alvo alcançada via televisão foi de $55 \%$; via rádio, $45 \%$; e via jornal, $80 \%{ }^{(13)}$.

Os participantes do grupo de intervenção que utilizaram o gráfico de risco, relatório de risco personalizado, ava-

\section{O vídeo educativo}

usou apelos

emocionais por

meio da narração

de histórias, com o

objetivo de aumentar

a motivação para

realização do exame

oftalmológico a cada

ano. Já o contato

telefônico guiado

por um protocolo

semiestruturado

oferecia educação

e aconselhamento

individualizado

e interativo, além

de identificar e

compreender as

razões de cada sujeito

e/ou barreiras para

realizar um exame de

fundo de olho ${ }^{(12)}$. liação da doença renal e da retinopatia e materiais suplementares de educação em gestão do diabetes receberam, em cada consulta, (1) avaliação do nível de HbAlc, pressão arterial e gravidade da RD; (2) gráfico personalizado, que foi mostrado para a visualização do risco de agravamento da $\mathrm{RD}$, com base no nível da HbAlc e no tipo de DM de cada indivíduo; (3) relatório personalizado resumindo o risco de acordo com a avaliação da doença renal e da RD, com base no nível de HbA1c; (4) gráfico plotagem dos níveis de $\mathrm{HbAlc}$ anteriores e atuais do participante; (5) e-mail com instruções para acessar achados de avaliação de risco individualizados; e (6) materiais suplementares de educação em gestão do diabetes ${ }^{(15)}$.

Os gráficos e relatórios personaliza$\operatorname{dos}^{(15)}$ não obtiveram êxito para o $\mathrm{al}$ cance do controle glicêmico, redução da HbAlc e melhora dos cuidados habituais com os olhos. Acredita-se que esse fato tenha decorrido da não adequação do material educativo à população-alvo, uma vez que possuíam características de difícil entendimento.

A adequação do material educativo aos indivíduos que vivenciam o problema nele abordado é uma atitude imprescindível, uma vez que possibilita a diminuição da distância entre o que foi exposto e o que foi apreendido, favorecendo o processo de ensino-aprendizagem.

A intervenção, mediante documentos impressos, teve como objetivo a ativação comportamental para a prevenção da $\mathrm{RD}$, combinando os princípios da educação sobre DM; a terapia comportamental e modelo de crença para ajudar os participantes a identificarem barreiras para a obtenção do exame de fundo de olho e, consequentemente, a superá-las; e a formulação de planos de ação para facilitar os exames. Os documentos possuíam três componentes: (1) etapas de realização de um exame oftalmológico (documento que lista atividades associadas ao agendamento e à obtenção do exame de fundo de olho); (2) passos para o controle glicêmico; (3) definição e principais carac- 
terísticas da RD, permitindo que os participantes refletissem sobre suas situações de vida ${ }^{(16)}$.

As intervenções educativas participativas, reflexivas e direcionadas ao público-alvo específico permitem potencializar práticas de aprendizagem autônoma e apresentam-se como instrumento didático e tecnológico, capaz de proporcionar conhecimento, favorecer a consciência crítica e promover saúde ${ }^{(18)}$. A intervenção educacional no cuidado de enfermagem é significante para o desenvolvimento de competências, pois permite e facilita a construção de conhecimentos e proporciona ao indivíduo a participação em um momento de troca de experiência, que corrobora o estímulo de habilidades e compreensão de determinados eventos ${ }^{(19)}$.

O público-alvo dos estudos foi formado por pessoas com DM dos tipos 1 ou 2, maiores de 18 anos, sem documentação de exame de fundo de olho. Os estudos $^{(12,14)}$ que utilizaram a intervenção telefônica incluíram apenas indivíduos com acesso a um telefone.

Estudos que tiveram a otimização da taxa dos cuidados habituais com os olhos realizados por diabéticos evidenciaram odds ratio para o status do exame de fundo de olho associado ao recebimento da intervenção variando de 2,5 - risco proporção de 2,58; intervalo de confiança de 95\% - IC95\% 1,91-3,48; $\mathrm{p}<0,001^{(16)}$ a 4,3 - IC95\% 2,4,-7,8; $\mathrm{p}<0,001^{(12)}$, corroborando a otimização da triagem da RD em $74 \%$ e reduzindo o risco de complicações oculares em uma população urbana pobre ${ }^{(14)}$.

O método usado para exames de retina depende dos recursos disponíveis e do nível de treinamento do profissional. $O$ rastreio ocular engloba acuidade visual (teste antes da dilatação da pupila) e exame de retina (pupilas dilatadas), como fotografia retiniana não midriática e/ou oftalmoscópio indireto binocular e/ou fotografia midriática da retina (câmara de fundo convencional) e/ou biomicroscopia de lâmpada de fenda. A dilatação da pupila pode melhorar a sensibilidade e a qualidade da imagem, especialmente quando os meios oculares não são claros, devido à catarata ${ }^{(20)}$.

Os cuidados habituais com os olhos incluem o controle do diabetes por meio de um estilo de vida saudável, suplementado com medicação, conforme necessário; exames oftalmológicos regulares; encaminhamento oportuno para o tratamento e o conhecimento dos sinais de alerta, como vista embaçada, perda de visão e distorção das imagens, porém os pacientes podem ser assintomáticos ${ }^{(3,20)}$.

As pessoas com DM precisam desempenhar papel ativo no gerenciamento de sua doença para evitar complicações que afetam sua qualidade de vida. Essa conquista pode ser auxiliada pelo enfermeiro com atividades de educação em saúde ${ }^{(20)}$.

Constatou-se, ainda, que o percentual de pessoas que realizou consulta com oftalmologista após 1 ano de intervenção educativa aumentou de $61 \%$ para $70 \%$ $(\mathrm{p}<0,001)$ e, após 2 anos, de $52 \%$ para $70 \%(\mathrm{p}<0,001)$; já a porcentagem de pessoas que notaram alteração na visão ao longo dos últimos 5 anos e vislumbraram algo sobre tal mudança aumentou levemente - de $91 \%$ para $93 \%{ }^{(13)}$.

Não foi estabelecida a duração ideal da intervenção educativa, uma vez que houve variação de 6 a 44 meses. Algumas intervenções com duração de 24 meses não mostraram resultados significativos, evidenciando que outras variáveis, como o tipo de intervenção, podem ser mais efetivas do que o tempo.

Quanto ao desfecho, a maioria dos estudos $^{(12-14,16)}$ relatou melhora significativa na realização dos exames de fundo de olho para a triagem da RD entre os pacientes com DM que participaram de intervenções educativas, evidenciando a importância dos exames oftalmológicos anuais para a saúde ocular das pessoas com DM.

Recomenda-se que o tempo de exames oftalmológicos iniciais e contínuos para a triagem da RD em pessoas com DM tipo 1 ocorra dentro de 5 anos após o diagnóstico e, para o DM tipo 2, o mais rapidamente possível após o diag- nóstico. Preconiza-se, ainda, um exame regular a cada 1 ou 2 anos se nenhuma anormalidade for detectada ${ }^{(20)}$.

Destaca-se, como limitação, a heterogeneidade entre os estudos, no que se refere a diferentes modos de aplicação da intervenção, o que impossibilitou a análise comparativa entre seus resultados.

Ante a complexidade da problemática do DM e da carência de conhecimento sobre a doença, ressalta-se a necessidade de mais estudos que corroborem a prevenção da RD e o rastreio de indivíduos assintomáticos, contribuindo para a otimização do diagnóstico e a prevenção de danos.

Ainda, merece destaque a carência de estudos realizados por enfermeiros, uma vez que tais profissionais desempenham papel importante no gerenciamento do diabetes e realizam ações capazes de otimizar o controle glicêmico e da pressão sanguínea e de lipídeos no sangue, para retardar a progressão da $\mathrm{RD}$, além de atuar na triagem, na educação e no apoio às pessoas com DM no controle de sua saúde ocular.

O cuidado clínico do enfermeiro possibilita ações ativas, como reflexão, análise crítica e resolução de problemas. A partir dessa compreensão, a educação da enfermagem mostra-se um instrumento de transformação da sociedade, cujas ações educativas realizadas no âmbito da educação em saúde ou permanente corroboram a qualidade da assistência prestada ao cliente.

Este estudo deve contribuir para o cuidado clínico de enfermagem a pacientes com DM na prevenção ou no controle da $\mathrm{RD}$, além de instigar o enfermeiro a realizar atividade de educação em saúde específica para cada público, assim como o desenvolvimento de estudos com bom nível de evidência.

\section{CONCLUSÃO}

A educação em saúde foi eficaz na prevenção e no controle da retinopatia diabética, porém não há consenso sobre as formas de intervenções. 
A disseminação de intervenções semelhantes, por meio do cuidado clínico de enfermagem, pode afetar significa- tivamente os indivíduos com diabetes mellitus em risco de retinopatia diabética e melhorar as disparidades de saúde existentes, bem como incorporar mais conhecimentos que fundamentem a ciência Enfermagem.

\section{REFERÊNCIAS}

1. American Diabetes Association (ADA). Standards of Medical Care in Diabetes - 2018. Diabetes Care [Internet]. 2018 [acesso em 19 Mar 2019]; 41(Suppl. 1):S1-S159. Disponivel em: https://diabetesed.net/wp-content/uploads/2017/12/2018-ADA-Standards-of-Care.pdf.

2. Henriques J, Vaz-Pereira S, Nascimento J, Rosa PC. Doença ocular diabética. Acta Med Port [Internet]. 2015 [acesso em 19 Mar 2019]; 28(1):107-13. Disponivel em: https://www.actamedicaportuguesa.com/revista/index.php/amp/article/viewFile/5361/4230.

3. Oliveira JP, Montenegro Junior RM, Vencio S, org. Diretrizes Sociedade Brasileira de Diabetes. 2017-2018 [Internet]. São Paulo: Clannad; 2017 [acesso em 19 Mar 2019]. Disponivel em: https://www.diabetes.org.br/profissionais/images/2017/diretrizes/diretrizes-sbd-2017-2018.pdf.

4. Barbosa L, Borges PC, Lemos SS, Cesarino CB. Avaliação da intervenção educativa em grupo para diabéticos assistidos em um Centro de Saúde Escola. Rev Enferm UERJ. 2016; 24(2):e4968.

5. Reis AE, Alaíde CM, Pacífico IR, Almeida CA, Rocha FC, Oliveira AD. Evidências da produção científica acerca da enfermagem na promoção do autocuidado em diabetes mellitus. R Interd [Internet]. 2017 [acesso em 19 Mar 2019]; 10(3):132-40. Disponivel em: https://revistainterdisciplinar.uninovafapi.edu. br/index.php/revinter/article/view/1276.

6. Souza LO, Figueiredo WS, Machado ML. As práticas de educação em diabetes vivenciadas no SUS: uma discussão da literatura com ênfase na atenção primária à saúde. Rev APS. 2017; 20(3):423-33.

7. Galvão TF, Pereira MG. Revisões sistemáticas da literatura: passos para sua elaboração. Epidemiol Serv Saúde [Internet]. 2014 [acesso em 19 Mar 2019]; 23(1):183-4. Disponivel em: http://scielo.iec.gov.br/scielo.php?script=sci_arttext\&pid=S1679-49742014000100018.

8. Galvão TF, Pansani TS, Harrad D. Principais itens para relatar Revisões sistemáticas e Meta-análises: A recomendação PRISMA. Epidemiol Serv Saúde. 2015; 24(2):335-42.

9. Malta M, Cardoso LO, Bastos FI, Magnanini MM, Silva CM. Iniciativa STROBE: subsídios para a comunicação de estudos observacionais. Rev Saúde Pública [Internet]. 2010 [acesso em 19 Mar 2019]; 44(3):559-65. Disponivel em: https://www. strobe-statement.org/fileadmin/Strobe/uploads/translations/STROBE_translation_portuguese_Commentary_Malta_RevSaudePublica_2010_checklist.pdf.

10. Jadad AR, Moore RA, Carrol D, Jenkinson C, Reynolds DM, Gavaghan DJ, et al. Assessing the quality freports of randomized clinical trials: is blinding necessary? Control Clin Trials. 1996; 17(1):1-12.
11. Downs SH, Black N. The feasibility of creating a checklist for the assessment of the methodological quality both of randomized and non-randomised studies of health care interventions. J Epidemiol Community Health. 1998; 52(6):377-84

12. Basch CE, Walker EA, Howard CJ, Shamoon H, Zybert P. The effect of health education on the rate of ophthalmic examinations among african americans with diabetes mellitus. Am J Public Health. 1999; 89(12):1878-82.

13. Walker EA, Schechter CB, Caban A, Basch CE. Telephone intervention to promote diabetic retinopathy screening among the urban poor. Am J Prev Med. 2008; 34(3):185-91

14. Aiello LP, Ayala AR, Antoszyk AN, Arnold-Bush B, Baker C, Bressler NM, Elman MJ, Glassman AR, Jampol LM, Melia M, Nielsen J, Wolpert HA. Diabetic Retinopathy Clinical Research Network. Assessing the effect of personalized diabetes risk assessments during ophthalmologic visitson glycemic control: a randomized clinical trial. JAMA Ophthalmol. 2015; 133(8):888-96.

15. Weiss DM, Casten RJ, Leiby BE, Hark LA, Murchison AP, Johnson D, et al. Effect of behavioral intervention on dilated fundus examination rates in older African American individuals with diabetes mellitus: a randomized clinical trial. JAMA Ophthalmol. 2015; 133(9):1005-12.

16. Müller A, Keeffe JE, Taylor HR. Changes in eye care utilization follow in ganeye health promotion campaign. Clin Exp Ophthalmol. 2007; 35(4):305-9.

17. Ministério da Saúde Secretaria de Ciência, Tecnologia e Insumos Estratégicos, Departamento de Ciência e Tecnologia (BR). Diretrizes metodológicas: Sistema GRADE - Manual de graduação da qualidade da evidência e força de recomendação para tomada de decisão em saúde. Brasília, DF: Ministério da Saúde, 2014.

18. Razera AP, Buetto LS, Lenza NF, Sonobe HM. Vídeo educativo; estratégias de ensino-aprendizagem para pacientes em tratamento quimioterápico. Ciênc Cuid Saúde [Internet]. 2014 [acesso em 19 Mar 2019]; 13(1):173-8. Disponivel em: http:// www.periodicos.uem.br/ojs/index.php/CiencCuidSaude/article/view/19659.

19. Berardinelli LM, Guedes NA, Ramos JP, Silva MG. Tecnologia educacional como estratégia de empoderamento de pessoas com enfermidades crônicas. Rev Enferm UERJ [Internet]. 2014 [acesso em 19 Mar 2019]; 22(5):603-9. Disponivel em: http://www.facenf.uerj.br/v22n5/v22n5a04.pdf.

20. International Diabetes Federation and The Fred Hollows Foundation. Diabetes eye health: A guide for health care professionals. Brussels, Belgium: International Diabetes Federation; 2015. 